\title{
A importância da Farmácia Universitária frente aos serviços clínicos prestados à comunidade
}

\author{
Bruna de Souza Vieira \\ Farmacêutica pelo Centro Universitário Católica de Quixadá \\ $\triangle$ bruna_010496@hotmail.com \\ Karla Bruna Nogueira Torres Barros \\ Mestre em Ensino na Saúde pela Universidade Estadual do Ceará \\ Leina Mércia de Oliveira Vasconcelos \\ Mestre em Saúde da Criança e do Adolescente pela Universidade Estadual do Ceará \\ Edilson Martins Rodrigues Neto \\ Doutor em Farmacologia Clínica pela Universidade Federal do Ceará \\ Maria Mayalle de Almeida Melo \\ Graduanda em Farmácia pelo Centro Universitário Católica de Quixadá \\ Sandna Larissa Freitas dos Santos \\ Residente em Saúde da Mulher e da Criança pela Universidade Federal do Ceará \\ Joelson Pinheiro de Lima \\ Graduando em Farmácia pelo Centro Universitário Católica de Quixadá
}

Recebido em 26 de junho de 2018

Aceito em 26 de novembro de 2018

\begin{abstract}
Resumo:
A Farmácia Universitária é um estabelecimento de saúde que desenvolve atividades de ensino, pesquisa e extensão nas áreas magistral e de dispensação de especialidades farmacêuticas, proporcionando aos acadêmicos do curso de farmácia a oportunidade de aliarem os conhecimentos teóricos adquiridos à prática diária das atividades do farmacêutico no exercício da profissão. 0 artigo tem como objetivo esclarecer os benefícios para a comunidade dos serviços disponibilizados pela farmácia universitária, juntamente com a atuação do Farmacêutico. Trata-se de uma revisão de literatura, na qual utilizou-se para realização da pesquisa as seguintes bases de dados: Pubmed, Bireme e Scielo, sendo selecionados após leitura dos resumos trabalhos completos de 2001 a 2017, perfazendo o total de 31 artigos. Nesse sentido, o farmacêutico assiste e orienta o paciente quanto a sua terapia medicamentosa, o mesmo atua na farmácia universitária que é uma farmácia comunitária que pertence a Instituição de Ensino, comportando atividades de consulta farmacêutica, seguimento farmacoterapêutico, dispensação de medicamentos, análise de água e manipulação de fitoterápicos. Os serviços disponibilizados devem atender as carências dos pacientes, orientando quanto a terapia farmacológica e não farmacológica dos mesmos. Desse modo, a farmácia universitária é um importante degrau para a formação acadêmica dos futuros profissionais, aliando ensino a prática, funcionando como um instrumento para a aproximação do profissional com a comunidade, refletindo no Uso Racional de Medicamentos, alcance de metas farmacoterapêuticas e na inclusão do mesmo junto a equipes multiprofissionais aliando conhecimentos e buscando a melhor efetivação dos serviços de saúde.
\end{abstract}

Palavras-chave: Assistência farmacêutica, Farmacoterapia, Farmácia Clínica. 


\title{
The importance of the University Pharmacy against the clinical services rendered to the community
}

\begin{abstract}
:
The University Pharmacy is a health establishment that develops teaching, research and extension activities in the master and dispensing areas of pharmaceutical specialties, providing pharmacy students with the opportunity to combine the theoretical knowledge acquired in the daily practice of the pharmacist's activities in the pharmacy exercise of the profession. The article aims to clarify the benefits to the community of the services offered by the university pharmacy, together with the Pharmacy's performance. It is a literature review, in which the following databases were used to perform the research: Pubmed, Bireme and Scielo, being selected after reading the abstracts complete works from 2001 to 2017, making a total of 31 articles. In this sense, the pharmacist assists and guides the patient regarding their drug therapy, he acts in the university pharmacy that is a community pharmacy that belongs to the Teaching Institution, including activities of pharmaceutical consultation, pharmacotherapeutic follow-up, dispensing of medicines, water analysis and manipulation of herbal medicines. The services provided should meet the needs of the patients, guiding them regarding pharmacological and non-pharmacological therapy of the same. Thus, university pharmacy is an important step for the academic training of future professionals, combining teaching and practice, functioning as an instrument for the professional approach to the community, reflecting on the Rational Use of Medications, the achievement of pharmacotherapeutic goals and the inclusion of it together with multiprofessional teams allying knowledge and seeking the best implementation of health services.
\end{abstract}

Keywords: Pharmaceutical Services, Drug Therapy, Clinical Pharmacy.

\section{La importancia de la Farmacia Universitaria frente a los servicios clínicos prestados à la comunidad}

\section{Resumen:}

La Farmacia Universitaria es un establecimiento de salud que desarrolla actividades de enseñanza, investigación científica y extensión en las áreas magistral y de dispensación de especialidades farmacéuticas, proponiendo a los académicos del curso de farmacia la oportunidad de aliar los conocimientos teóricos adquiridos a la práctica diaria de las actividades del farmacéutico en ejercicio de la profesión. El artículo tiene como objetivo aclarar los beneficios para la comunidad a través de los servicios ofrecidos por la farmacia universitaria, junto con la actuación del Farmacéutico. Se trata de una revisión de literatura, en la que se utilizó para realizar la investigación las siguientes bases de datos: Pubmed, Bireme y Scielo, siendo seleccionados después de la lectura de los resúmenes trabajos completos de 2001 a 2017, totalizando el total de 31 artículos. En este sentido, el farmacéutico asiste y orienta al paciente en cuanto a su terapia medicamentosa, el mismo actúa en la farmacia universitaria que es una farmacia comunitaria que pertenece a la Institución de Enseñanza que contiene actividades de consulta farmacéutica, seguimiento farmacoterapéutico, dispensación de medicamentos, análisis de agua y la manipulación de los fitoterápicos. Los servicios disponibles deben atender las carencias de los pacientes, orientando en cuanto a terapia farmacológica y no farmacológica de los mismos. De este modo, la farmacia universitaria es un importante escalón para la formación académica de los futuros profesionales, aliando la enseñanza de la práctica, funcionando como un instrumento para la aproximación del profesional con la comunidad, contribuyendo con el Uso Racional de Medicamentos, alcance de metas farmacoterapéuticas y en la inclusión del mismo junto a equipos multiprofesionales aliando conocimientos y buscando la mejor efectividad de los servicios de salud.

Palabras clave: Servicios farmacéuticos, Tratamiento Farmacológico, Farmacia Clínica. 


\section{INTRODUÇÃo}

A Farmácia Universitária é um estabelecimento de saúde, vinculado ao ensino superior, que se destina a aprimorar os acadêmicos e os profissionais farmacêuticos, através de atividades e serviços que são disponibilizados à comunidade de acordo com a necessidade de cada paciente, proporcionando assim uma assistência farmacêutica adequada a quem precisa (PIMENTA, 2010).

A finalidade da farmácia universitária é o fornecimento de informações para os pacientes sobre como utilizar os medicamentos de forma racional, através de um atendimento especializado e humanizado durante a consulta farmacêutica e dispensação de medicamentos, da mesma forma instruir os usuários sobre a forma correta de manter os medicamentos em casa, armazenando-os corretamente e como descarta-los de maneira adequada quando for necessário. Para o acadêmico neste estabelecimento será mostrado a realidade do seu futuro local de trabalho, bem como a oportunidade de desenvolvimento social junto à comunidade (MIYOSHI; BAGLIE, 2014).

A consulta farmacêutica é um marco importante para a farmácia universitária, pois será através deste serviço que o farmacêutico irá realizar a atenção e assistência necessária para garantia da eficácia terapêutica, do tratamento da doença e acima de tudo da manutenção da saúde e da qualidade de vida, a resolução no 585 de 29 de agosto de 2013, regulamenta as atribuições clínicas do farmacêutico e dá outras providências, de acordo com esta resolução a consulta farmacêutica deve ser realizada em um consultório farmacêutico ou em outro local adequado (RUSTON; SILVA, 2011; CONSELHO FEDERAL DE FARMÁCIA, 2013).

Diante da importância de um acompanhamento, o farmacêutico se torna um profissional de grande relevância para os pacientes, pois do ponto de vista da saúde pública, as farmácias são locais de grande busca por atendimento e possível porta de entrada dos pacientes no sistema de saúde, então é importante que o farmacêutico aconselhe os seus pacientes, interaja e discuta as necessidades de cada pessoa atendida, de forma que cada atendimento seja único e individualizado (SILVA; NAVES; VIDAL, 2008; DADER; MUNÕZ; MARTINEZ, 2007). 
A atenção farmacêutica é uma prática sugerida e desenvolvida dentro da profissão farmacêutica ao longo da década de 90 . Desde a sua idealização esta prática foi aceita como nova tarefa dos farmacêuticos por organizações de apoio e gestão de saúde, órgãos farmacêutico e faculdade de farmácia em vários lugares do mundo, sendo que a criação deste modelo profissional aconteceu devido à necessidade social referente à grande prevalência de morbidade e mortalidade pelo uso incorreto de medicamentos, estabelecendo um sério problema de saúde coletiva (MACHADO et al., 2004).

É importante que haja interação significativa entre paciente e o farmacêutico, pois durante a dispensação do medicamento a orientação correta é imprescindível para que este utilize o medicamento de forma racional e segura. No momento da consulta o farmacêutico deve atentar-se para as solicitações e queixas do paciente, escutando-o prontamente e realizando de forma sistemática e documentada a consulta farmacêutica, especializada principalmente na escuta permanente e padronizada (PRUDENTE et al., 2012).

Os serviços que serão disponibilizados deverão ultrapassar as fronteiras dos medicamentos, promovendo um atendimento mais inclusivo, focando na qualidade de vida dos pacientes e interações humanas (PIMENTA, 2010).

Logo, os serviços disponibilizados por farmácias universitárias, auxiliam de forma benéfica a comunidade através da prescrição de medicamentos necessários, evitando complicações devido ao uso incorreto destes, reduzindo ainda a automedicação. Partindo deste ponto, o estudo faz-se relevante tendo-se em vista que diversos fatores ainda ocasionam terapias medicamentosas inadequadas aos pacientes, como Problemas Relacionados à Medicamentos (PRM's), interações medicamentosas e reações adversas. Sendo fundamental a inserção do farmacêutico, auxiliando e intervindo quando necessário, buscando a efetividade farmacoterapêutica e qualidade de vida do paciente. Sendo um trabalho de relevância acadêmica e social, reportando o aperfeiçoamento do aprendizado e a promoção de saúde aos pacientes.

Com base nisso, o estudo objetiva-se a esclarecer os benefícios para a comunidade através dos serviços disponibilizados pela farmácia universitária, juntamente com a atuação do Farmacêutico. 


\section{METODOLOGIA}

A metodologia empregada foi uma revisão de literatura. A Identificação dos artigos foi feita através da busca nos bancos de dados informatizados Pubmed (National Library of Medicine National Institutes of Health), Bireme (Biblioteca Virtual em Saúde) e Scielo (Biblioteca eletrônica Scientific Electronic Library Online) de 2001 a 2017. As palavras-chaves utilizadas na identificação dos artigos foram: "assistência farmacêutica", "farmacoterapia" e "farmácia clínica” e suas traduções para língua inglesa.

Os critérios de inclusão para a seleção dos artigos foram: artigos originais de pesquisa sobre os serviços disponibilizados pela farmácia universitária, publicados em português, espanhol e inglês, bem como revisões da literatura dissertações e teses. Os critérios de exclusão foram: artigos não pesquisados no Brasil, editoriais, relatos de casos, estudos apresentados em Congressos e Seminários, e publicados em anos anteriores.

Os resumos foram avaliados, e realizado a leitura das produções que atenderam os critérios estabelecidos previamente e que foram selecionadas para este estudo. Organizou-se a temática em: A importância da farmácia universitária, os benefícios para a comunidade dos serviços disponibilizados pela farmácia universitária e a importância do Farmacêutico na farmácia comunitária.

Obteve-se um total de 38 publicações relacionados ao tema, sendo que apenas 31 se esquadravam dentro dos critérios de inclusão. Destes 29 (93,55\%) eram descritos em português, 1 (3,22\%) em inglês e 1 (3,22\%) em espanhol. Quanto ao período de publicação, constatou-se que os anos que apresentaram maior número de artigos publicados foram 2012, sendo que nos últimos três anos obteve: dois artigos em 2015 (6,44\%), 2016 com dois $(6,44 \%)$ e 2017 com uma (3,22\%) publicação. 


\section{REVISÃO DE LITERATURA}

\section{A importância do farmacêutico atuando na farmácia comunitária}

A sociedade ainda vislumbra a farmácia apenas como um estabelecimento de obtenção de medicamentos sob boas condições, mas esse não é o único serviço das farmácias, este é um local onde deve-se dispensar medicamentos de forma correta, realizar serviços clínicos e cabe ao farmacêutico executar esses serviços caracterizando, assim, a farmácia como um estabelecimento que agrega dimensões comerciais, sanitárias, técnicas e social, pois este se caracteriza como um estabelecimento de saúde (CORRER; OTUKI, 2013).

De acordo com Furtado (2001), o farmacêutico é um profissional que assiste e orienta diretamente o paciente quanto a sua terapia medicamentosa prescrita pelo médico, e este busca sempre o melhor para esses pacientes, analisando as necessidades e os problemas que podem surgir. Para que esta assistência seja dada de forma correta, o profissional deve estar apto, dominando o conhecimento necessário, empenhado e com responsabilidade nas suas ações, assim o profissional vai conquistar o seu devido reconhecimento diante da sociedade (OLIVEIRA, 2005).

A resolução nº 499 de 17 de dezembro de 2008, dispõe sobre a prestação de serviços farmacêuticos, em farmácias e drogarias, e dá outras providências, por meio desta resolução fica estabelecido que somente o farmacêutico inscrito no Conselho Regional de Farmácia de sua jurisdição, poderá prestar serviços farmacêuticos em farmácias e drogarias (CONSELHO FEDERAL DE FARMÁCIA, 2008).

De acordo com a Lei ํㅡㄴ 13.021 de 8 de agosto de 2014, as farmácias comunitárias serão classificadas segundo a sua natureza, como: farmácia sem manipulação ou drogaria e farmácia com manipulação e por meio desta lei confirmou-se a farmácia como um estabelecimento de saúde, onde o farmacêutico deve prestar os seus serviços (BRASIL, 2014).

Antes desta lei, já eram permitidos na farmácia alguns serviços, incluindo: atenção farmacêutica (atenção farmacêutica domiciliar, a aferição de parâmetros fisiológicos e bioquímico e a administração de medicamentos) e perfuração de lóbulo auricular para colocação de brincos. Porém, com o advento da norma, esse rol foi ampliado, assim o farmacêutico também pode trabalhar com disponibilidade imediata de vacinas e soros conforme o perfil epidemiológico da região (CONSELHO REGIONAL DE FÁRMACIA, 2015). 
O Farmacêutico é um profissional essencial para uma farmacoterapia significativa. Vale ressaltar que a intervenção farmacêutica pode prevenir algumas complicações que possam surgir com a utilização de medicamentos, estas ações de interposições podem estar relacionadas com a dose do medicamento (adequada ou inadequada), a forma de apresentação, horário e frequência, a partir dessas ações o farmacêutico contribui positivamente para o bem-estar do assistido (MIRANDA et al., 2012).

\section{Farmácia universitária}

A farmácia universitária é uma farmácia comunitária que pertence a IES e pode estar localizada no campus da instituição, sendo o principal objetivo desse estabelecimento proporcionar aos estudantes conhecimento através de vivências profissionais, como também, disponibilizar serviços à comunidade interna e externa da IES. As atividades realizadas na farmácia universitária podem se restringir apenas a uma área ou podem oferecer serviços de diversas áreas de forma integrada (ROSSIGNOLI; CORRER; FERNÁNDEZ-LLIMÓS, 2003).

Trata-se de um local que tem como objetivo o aprendizado dos acadêmicos de farmácia por meio das situações profissionais vivenciadas por estes no cotidiano e oferecer serviços farmacêuticos para a comunidade respeitando as legislações sanitárias, com compromisso ético, trabalho em equipe e tomada de decisões, proporcionando aos pacientes/clientes um serviço de qualidade e responsável (BATISTA, 2012).

De modo geral a farmácia universitária, de acordo com a Agência Nacional de Vigilância Sanitária (ANVISA) é uma das modalidades da farmácia comunitária que deve auxiliar a comunidade através dos serviços que são oferecidos por este estabelecimento, prestando de forma benéfica, atendimento de qualidade para os pacientes/clientes acolhidos e acompanhados. É um estabelecimento de saúde, que busca o intercâmbio entre a teoria, os problemas sociais, a prática e o aprendizado (SATURNINO; FERNANDÉZ-LLIMÓS, 2009).

Tal estabelecimento atua como instrumento de pesquisa e extensão, tem como propósito a formação dos estudantes de farmácia e o aperfeiçoamento do profissional farmacêutico, auxiliando com a prestação de serviços farmacêutico e com a dispensação de medicamentos industrializados ou manipulados, contribuindo deste modo para a proteção, promoção e recuperação da saúde. 
De acordo com a Diretriz Curricular Nacional do curso de graduação em farmácia DCN, a farmácia universitária agora se torna um local obrigatório de prática, que pode estar localizada na IES ou em outro estabelecimento, que esteja envolvido à assistência farmacêutica, mediante convênio, objetivando à aplicação de atividades de estágio obrigatório, para todos os acadêmicos do curso, visando uma formação pautada em princípios éticos e científicos, capacitando-o para o trabalho nos diferentes níveis de complexidade do sistema de saúde (MINISTÉRIO DA EDUCAÇÃO, 2017).

Nesse sentido, de acordo com resolução n⿳ 610, de 25 de março de 2015, que dispõe sobre as atribuições do Farmacêutico na Farmácia Universitária, o mesmo deve realizar o serviço de cuidado farmacêutico ao paciente, a família e a comunidade, executar a dispensação de medicamentos industrializados ou magistrais, fornecer informações sobre plantas medicinais, drogas vegetais e outras categorias, com o objetivo do uso racional de medicamentos e uma melhor farmacoterapia (CONSELHO FEDERAL DE FARMÁCIA, 2015).

A farmácia universitária, de acordo com a legislação, é um estabelecimento que visa formar profissionais qualificados, além disso é uma unidade que ajuda a população atendida, através de serviços destinados a fornecer assistência farmacêutica, assistência à saúde e orientação sanitária individual e coletiva, sendo um local de grande procura pela população que necessita do serviço de atenção farmacêutica (POSSAGNO et al., 2016).

Os serviços disponibilizados pela farmácia universitária (Quadro 1) tem como objetivo ajudar de forma benéfica toda a comunidade assistida. Um dos serviços é a atenção farmacêutica, que assim como outras práticas profissionais, sugere uma metodologia de trabalho ou método de cuidado do paciente, sendo que esta prática prevê uma abordagem centrada no indivíduo, a construção de um vínculo terapêutico entre farmacêutico e paciente e o compromisso profissional em assegurar que todas as necessidades farmacoterapêuticas dos pacientes serão atendidas (MACHADO et al., 2004). 
Quadro 1 - Serviços disponibilizados pela farmácia universitária

\begin{tabular}{|c|c|c|c|c|c|}
\hline $\begin{array}{l}\text { ARTIGO/ } \\
\text { AUTORES }\end{array}$ & PERIÓDICO & ANO & TIPO DE PESQUISA & OBJETIVO & RESULTADO \\
\hline $\begin{array}{l}\text { Resolução N } \\
585 \text { de } 29 \text { de } \\
\text { Agosto de } \\
2013 / \\
\text { CONSELHO } \\
\text { FEDERAL DE } \\
\text { FARMÁCIA }\end{array}$ & $\begin{array}{l}\text { Diário Oficial } \\
\text { da União }\end{array}$ & 2013 & $\begin{array}{l}\text { Resolução do Conselho } \\
\text { Federal de Farmácia }\end{array}$ & $\begin{array}{l}\text { Regulamenta as } \\
\text { atribuições clínicas } \\
\text { do farmacêutico e dá } \\
\text { outras providências. }\end{array}$ & $\begin{array}{l}\text { Consulta } \\
\text { Farmacêutica: } \\
\text { A resolução n } 585 \\
\text { de } 29 \text { de agosto de } \\
2013 \text {, prover este } \\
\text { serviço, de forma } \\
\text { que a consulta } \\
\text { farmacêutica busca } \\
\text { promover o uso } \\
\text { racional de } \\
\text { medicamentos, é } \\
\text { um serviço único e } \\
\text { o profissional que } \\
\text { for realizá-lo deve } \\
\text { se especializar nos } \\
\text { problemas mais } \\
\text { comuns da região, } \\
\text { o farmacêutico } \\
\text { deve conhecer o } \\
\text { perfil } \\
\text { epidemiológico e as } \\
\text { necessidades dos } \\
\text { pacientes do local } \\
\text { no qual está } \\
\text { inserido. }\end{array}$ \\
\hline $\begin{array}{l}\text { Método } \\
\text { Dáder. Manual } \\
\text { de seguimento } \\
\text { farmacoterap } \\
\text { êutico / } \\
\text { HERNÁNDEZ, } \\
\text { D.S.; CASTRO, } \\
\text { M.M.S.; } \\
\text { DÁDER, M.J.F. }\end{array}$ & $\begin{array}{l}\text { Edições } \\
\text { Universitárias } \\
\text { Lusófonas. } \\
\text { Terceira } \\
\text { Edição }\end{array}$ & 2010 & $\begin{array}{l}\text { Guia de Seguimento } \\
\text { Farmacoterapêutico }\end{array}$ & $\begin{array}{l}\text { Direcionar e } \\
\text { explanar formas de } \\
\text { abordagens com } \\
\text { relação a consulta } \\
\text { farmacêutica }\end{array}$ & $\begin{array}{l}\text { Seguimento } \\
\text { Farmacoterapêuti } \\
\text { co: } \\
\text { É utilizado para } \\
\text { conseguir os } \\
\text { melhores } \\
\text { resultados na } \\
\text { terapia dos } \\
\text { pacientes, } \\
\text { assegurando a } \\
\text { efetividade e } \\
\text { segurança dos } \\
\text { medicamentos. } \\
\text { Neste seguimento o } \\
\text { profissional tenta } \\
\text { detectar algum } \\
\text { problema } \\
\text { relacionado a } \\
\text { medicamento } \\
\text { (PRM), tentando } \\
\text { prevenir e resolver } \\
\text { os resultados } \\
\text { negativos que } \\
\text { podem aparecer } \\
\text { com a utilização de } \\
\text { medicamentos, este } \\
\text { é um serviço que } \\
\text { deve ser contínuo, } \\
\text { sistemático e }\end{array}$ \\
\hline
\end{tabular}




\begin{tabular}{|c|c|c|c|c|c|}
\hline & & & & & $\begin{array}{l}\text { documentado, com } \\
\text { a colaboração dos } \\
\text { demais } \\
\text { profissionais de } \\
\text { saúde e com o } \\
\text { paciente para que } \\
\text { juntos escolham a } \\
\text { melhor forma de se } \\
\text { obter os resultados } \\
\text { positivos da } \\
\text { farmacoterapia }\end{array}$ \\
\hline $\begin{array}{l}\text { A dispensação } \\
\text { de } \\
\text { medicamentos } \\
\text { :uma reflexão } \\
\text { sobre o } \\
\text { processo para } \\
\text { prevenção, } \\
\text { identificação e } \\
\text { resolução de } \\
\text { problemas } \\
\text { relacionados à } \\
\text { farmacoterapi } \\
\text { a / } \\
\text { GALATO, D.; } \\
\text { ALANO, G.M.; } \\
\text { TRAUTHMAN, } \\
\text { S.C.; VIEIRA, } \\
\text { A.C. }\end{array}$ & $\begin{array}{l}\text { Revista } \\
\text { Brasileira de } \\
\text { Ciências } \\
\text { Farmacêuticas }\end{array}$ & 2008 & $\begin{array}{l}\text { Pesquisa qualitativa } \\
\text { baseada em pesquisa- } \\
\text { ação }\end{array}$ & $\begin{array}{l}\text { Apresentar uma } \\
\text { reflexão sobre a } \\
\text { dispensação de } \\
\text { medicamentos na } \\
\text { farmácia comunitária }\end{array}$ & $\begin{array}{l}\text { Dispensação de } \\
\text { medicamentos: } \\
\text { É um dos processos } \\
\text { de atenção à saúde } \\
\text { ao paciente, deve } \\
\text { ser um processo } \\
\text { realizado em } \\
\text { conjunto com todos } \\
\text { os profissionais, } \\
\text { principalmente } \\
\text { com os } \\
\text { prescritores. A } \\
\text { dispensação } \\
\text { começa no } \\
\text { momento da } \\
\text { análise da } \\
\text { prescrição, } \\
\text { identificando o } \\
\text { paciente que está } \\
\text { recebendo o } \\
\text { atendimento, deve- } \\
\text { se analisar se o } \\
\text { paciente faz uso de } \\
\text { algum } \\
\text { medicamento, se } \\
\text { este já teve contato } \\
\text { com algum } \\
\text { medicamento, } \\
\text { inclusive aquele } \\
\text { que vai ser } \\
\text { dispensando, o } \\
\text { profissional deve } \\
\text { realizar as devidas } \\
\text { orientaçães de } \\
\text { como utilizar o } \\
\text { medicamento } \\
\text { tentando buscar a } \\
\text { efetividade e } \\
\text { segurança da } \\
\text { terapia } \\
\text { medicamentosa }\end{array}$ \\
\hline $\begin{array}{l}\text { Resolução no } \\
463 \text {, de } 27 \text { de } \\
\text { junho de } 2007 \\
\text { / CONSELHO } \\
\text { FEDERAL DE } \\
\text { FARMÁCIA }\end{array}$ & $\begin{array}{l}\text { Diário Oficial } \\
\text { da União }\end{array}$ & 2007 & $\begin{array}{l}\text { Resolução do Conselho } \\
\text { Federal de Farmácia }\end{array}$ & $\begin{array}{l}\text { Dispõe sobre as } \\
\text { atribuições do } \\
\text { Farmacêutico no } \\
\text { controle de } \\
\text { qualidade e } \\
\text { tratamento de água } \\
\text { para consumo }\end{array}$ & $\begin{array}{l}\text { Análise de água: } \\
\text { De acordo com a } \\
\text { resolução no } 463 \text {, de } \\
27 \text { de junho de } 2007 \\
\text { são atribuições do } \\
\text { farmacêutico a } \\
\text { análise e o controle }\end{array}$ \\
\hline
\end{tabular}




\begin{tabular}{|c|c|c|c|c|c|}
\hline & & & & $\begin{array}{l}\text { humano, seu padrão } \\
\text { de potabilidade e } \\
\text { controle ambiental, } \\
\text { bem como o controle } \\
\text { de operação das } \\
\text { estações de } \\
\text { tratamento de água e } \\
\text { esgotos domésticos e } \\
\text { industriais, de } \\
\text { piscinas, praias, } \\
\text { balneários, hotéis, } \\
\text { condomínios e } \\
\text { congêneres. }\end{array}$ & $\begin{array}{l}\text { de águas minerais e } \\
\text { residuárias, para o } \\
\text { uso humano, em } \\
\text { todas as formas e } \\
\text { padrão de } \\
\text { potabilidade }\end{array}$ \\
\hline $\begin{array}{l}\text { Formulário } \\
\text { nacional da } \\
\text { farmacopeia } \\
\text { brasileira / } \\
\text { Ministério da } \\
\text { Saúde. } \\
\text { Agência } \\
\text { Nacional de } \\
\text { Vigilância } \\
\text { Sanitária. }\end{array}$ & $\begin{array}{l}\text { Ministério da } \\
\text { Saúde. }\end{array}$ & 2012 & $\begin{array}{l}\text { Formulário/Compêndi } \\
\text { o }\end{array}$ & $\begin{array}{l}\text { Direcionar farmácias } \\
\text { de manipulação e } \\
\text { Centros } \\
\text { Universitários de } \\
\text { formação para o } \\
\text { seguimento de } \\
\text { manipulação de } \\
\text { produtos } \\
\text { farmacêuticos }\end{array}$ & $\begin{array}{l}\text { Manipulação de } \\
\text { fitoterápicos e } \\
\text { medicamentos: } \\
\text { A RDC no } 67, \text { de } \\
\text { outubro de } 2007 \text {, } \\
\text { dispõe sobre as } \\
\text { Boas Práticas de } \\
\text { Manipulação de } \\
\text { preparações } \\
\text { magistrais e } \\
\text { oficinais para uso } \\
\text { humano em } \\
\text { farmácias, os } \\
\text { produtos }\end{array}$ \\
\hline
\end{tabular}




\begin{tabular}{|c|c|c|c|c|c|}
\hline $\begin{array}{l}\text { Resolução no } \\
67, \text { de } 8 \text { de } \\
\text { outubro de } \\
2007 .\end{array}$ & $\begin{array}{l}\text { Diário Oficial } \\
\text { da União }\end{array}$ & 2007 & $\begin{array}{l}\text { Resolução da Diretoria } \\
\text { Colegiada da Agência } \\
\text { Nacional de Vigilância } \\
\text { Sanitária }\end{array}$ & $\begin{array}{l}\text { Fixa os requisitos } \\
\text { mínimos exigidos } \\
\text { para o exercício das } \\
\text { atividades de } \\
\text { manipulação de } \\
\text { preparações } \\
\text { magistrais e oficinais } \\
\text { das farmácias, desde } \\
\text { suas instalações, } \\
\text { equipamentos e } \\
\text { recursos humanos, } \\
\text { aquisição e controle } \\
\text { da qualidade da } \\
\text { matéria-prima, } \\
\text { armazenamento, } \\
\text { avaliação } \\
\text { farmacêutica da } \\
\text { prescrição, } \\
\text { manipulação, } \\
\text { fracionamento, } \\
\text { conservação, } \\
\text { transporte, } \\
\text { dispensação das } \\
\text { preparações, além da } \\
\text { atenção farmacêutica } \\
\text { aos usuários ou seus } \\
\text { responsáveis, } \\
\text { visando à garantia de } \\
\text { sua qualidade, } \\
\text { segurança, } \\
\text { efetividade e } \\
\text { promoção do seu uso } \\
\text { seguro e racional. }\end{array}$ & $\begin{array}{l}\text { manipulados são } \\
\text { aqueles obtidos em } \\
\text { farmácias } \\
\text { aplicando-se estas } \\
\text { boas práticas, de } \\
\text { acordo com a } \\
\text { prescrição de um } \\
\text { profissional } \\
\text { habilitado ou } \\
\text { indicação pelo } \\
\text { farmacêutico. A } \\
\text { qualidade dos } \\
\text { produtos } \\
\text { manipulados } \\
\text { depende do } \\
\text { conhecimento } \\
\text { científico, da } \\
\text { capacitação } \\
\text { profissional e } \\
\text { competência } \\
\text { técnica do } \\
\text { farmacêutico e da } \\
\text { contínua interação } \\
\text { entre farmacêutico, } \\
\text { prescritor e } \\
\text { paciente. Porém } \\
\text { também é } \\
\text { importante a } \\
\text { organização e } \\
\text { controle do } \\
\text { farmacêutico sobre } \\
\text { as técnicas de } \\
\text { preparo, matérias- } \\
\text { primas, } \\
\text { equipamentos e } \\
\text { instrumentos } \\
\text { utilizados, } \\
\text { condições de } \\
\text { armazenamento } \\
\text { dos insumos e } \\
\text { produtos e da } \\
\text { documentação } \\
\text { sobre todos os } \\
\text { procedimentos, } \\
\text { materiais e } \\
\text { recursos } \\
\text { empregados na } \\
\text { preparação dos } \\
\text { produtos } \\
\text { magistrais em } \\
\text { todas as suas fases, } \\
\text { incluindo aquelas } \\
\text { posteriores à } \\
\text { dispensação }\end{array}$ \\
\hline
\end{tabular}

Fonte: Scientific Electronic Library (SCIELO), Agência Nacional de Vigilância Sanitária (ANVISA), Ministério da Saúde e Conselho Federal de Farmácia (CFF) - anos de 2007 a 2013. 
Ainda com relação ao Quadro 1, é importante que relate-se a importância de alguns serviços disponibilizados pelas farmácias universitária, como no caso da análise de água, este procedimento é essencial, tendo-se em vista que a contaminação da água pode ser responsável por uma grande número de doenças de veiculação hídrica, principalmente em regiões onde as situações de saneamento são precárias, podem surgir doenças, por exemplo, diarreicas, febre tifoide, cólera, verminoses, hepatite A. Devido a essa contaminação da água podem surgir alguns surtos epidêmicos, que podem levar a altas taxas de mortalidade infantil, por isso a análise da água é de extrema importância para à comunidade, pois se a qualidade da água utilizada é verificada pode-se evitar possíveis doenças decorrentes dessa água contaminada (FREITAS; BRILHANTE; ALMEIDA, 2001).

Com relação ao método Dáder, este funciona como uma das ferramentas do seguimento farmacoterapêutico baseando-se na obtenção da história farmacoterapêutica do paciente, buscando os problemas de saúde que ele apresenta e os medicamentos que utiliza, avaliando o estado do utente afim de identificar e resolver os possíveis PRM's que podem surgir. Após a identificação, se realizarão as intervenções farmacêuticas necessárias para resolver os PRM's e posteriormente se avaliarão os resultados obtidos (MACHUCA; FERNANDEZ-LLIMÓS; FAUS, 2003).

\section{CONSIDERAÇÕES FINAIS}

A farmácia universitária e os serviços disponibilizados por esta são de grande relevância para o desenvolvimento inicial e avanço da prática de assistência farmacêutica, sendo um estabelecimento de saúde, comprometida com as causas importantes da profissão e com a necessidade de contribuir através da prática clínica com a qualidade de vida dos pacientes atendidos.

A mesma funciona como um local de ensino, proporcionando aos discentes a aplicação e ampliação deste junto à comunidade, por meio do que se chama de extensão. Os serviços clínicos obtidos junto à farmácia universitária funcionam como um adendo aos novos profissionais, adicionando experiência clínica, bem como a oportunidade de aplicar conhecimentos adquiridos durante o percurso da graduação, funcionando ainda como um 
importante pilar da construção de conhecimento da população a respeito de atividades desenvolvidas por farmacêuticos e da relação do profissional com a comunidade.

Nesse sentido, a implantação de farmácias universitárias junto aos cursos de graduação em farmácia é fundamental no que compete a formação dos discentes, sendo que a Diretriz Curricular Nacional do curso de graduação em farmácia (DCN), admite que tal estabelecimento deve circular entre os campos de estágio obrigatório durante a formação acadêmica, sendo essencial a adequação das IES à essa nova realidade.

Dessa forma o presente estudo permite fornecer informações a respeito dos serviços clínicos que podem ser realizados por acadêmicos e profissionais inseridos no contexto da farmácia universitária, tendo como arcabouço as resoluções específicas que fundamentam tais atividades. Proporcionando ainda, o conhecimento por parte de outros profissionais de saúde e da população em geral a respeito dos objetivos e benefícios do cuidado farmacêutico. Sendo de fundamental importância, frente ao atual cenário do país, tendo-se em vista que a aproximação da comunidade com o farmacêutico, contribui para a melhoria da assistência em saúde, esclarecendo-os a respeito do uso de medicamentos, bem como riscos e benefícios, refletindo por conseguinte na redução de gastos em saúde devido ao uso não racional dos mesmos por parte da população e na efetividade da realização do cuidado em saúde.

\section{REFERÊNCIAS}

BATISTA, J. L. A implantação da farmácia-escola: o desafio de uma parceria público privada na melhoria da gestão da assistência farmacêutica de Lajeado, RS. 2012. 33f. Trabalho de Conclusão de Curso (Especialização) - Escola de Administração, Universidade Federal do Rio Grande do Sul, Porto Alegre, 2012.

BRASIL. Ministério da Saúde. Agência Nacional de Vigilância Sanitária. Formulário nacional da farmacopeia brasileira / Brasil. $2^{\mathrm{a}}$ edição. Brasília: Anvisa, 2012. 224 p.

. Lei 13.021 de 8 de agosto de 2014. Dispõe sobre o exercício e a fiscalização das atividades farmacêuticas.

CONSELHO FEDERAL DE FARMÁCIA. Dispõe sobre a prestação de serviços farmacêuticos, em farmácias e drogarias, e dá outras providências. Resolução nº 499, de 17 de dezembro de 2008. Lex: Diário Oficial da União, 2008.

Dispõe sobre as atribuições do farmacêutico na farmácia universitária e dá outras providências. Resolução nº 610, de 20 de março de 2015. Lex: Reunião Plenária SHIS QI 15 Lote L. Lago Sul, Brasília, 2015.

. Dispõe sobre Boas Práticas de Manipulação de Preparações Magistrais e Oficinais para Uso Humano em farmácias. Resolução nº 67, de 8 de outubro de 2007. Lex: Diário Oficial da União, 2007. 
. Ementa: Dispõe sobre as atribuições do Farmacêutico no controle de qualidade e tratamento de água para consumo humano, seu padrão de potabilidade e controle ambiental, bem como o controle de operação das estações de tratamento de água e esgotos domésticos e industriais, de piscinas, praias, balneários, hotéis, condomínios e congêneres. Resolução nº 463, de 27 de junho de 2007. Lex: Diário Oficial da União, 2007.

. Ementa: Regula a prescrição farmacêutica e dá outras providências. Resolução nº 586, de 29 de agosto de 2013. Lex: Diário Oficial da União, 2013.

CORRER, C.J.; OTUKI, M.F. A prática farmacêutica na farmácia comunitária. Artmed. Porto Alegre, 2013.

. Método clínico de atenção farmacêutica. Revista Pan -Amazônica de Saúde. Março, 2011.

DADER, M.J.; MUNÕZ, P.A.; MARTÍNEZ, F. Atenção Farmacêutica: conceitos, processos e casos práticos. Tradução e revisão de Maria Denise Funchal Witzel. São Paulo. RCN Editora, 2007.

FREITAS, M.B.; BRILHANTE, O.M., ALMEIDA, L.M. Importância da análise de água para a saúde pública em duas regiões do Estado do Rio de Janeiro: enfoque para coliformes fecais, nitrato e alumínio. Caderno de Saúde Pública. Vol.17, n. 3, p. 651-660, maio-junho. Rio de Janeiro, 2001.

FURTADO, G. R. Noções Básicas sobre Atenção Farmacêutica. Curitiba: Editora UFPR, 2001. 23 p.

GALATO, D.; ALANO, G.M.; TRAUTHMAN, S.C.; VIEIRA, A.C. A dispensação de medicamentos: uma reflexão sobre o processo para prevenção, identificação e resolução de problemas relacionados à farmacoterapia. Revista Brasileira de Ciências Farmacêuticas. Vol. 44, n. 3, jul./set. Universidade do Sul de Santa Catarina, 2008.

HERNÁNDEZ, D.S.; CASTRO, M.M.S.; DÁDER, M.J.F. Método Dáder. Manual de seguimento farmacoterapêutico. Edições Universitárias Lusófonas. Terceira Edição - Versão em Português Europeu. Lisboa, 2010.

MACHADO, R.M.C.; FREITAS, E.L.; PEREIRA, M.L.; OLIVEIRA, D.R. Implementação da Atenção Farmacêutica na Farmácia Universitária da UFMG. Anais do $2^{\text {o }}$ Congresso Brasileiro de Extensão Universitária. Belo Horizonte, 12 a 15 de setembro de 2004.

MACHUCA, M.; FERNÁNDEZ-LLIMÓS, F.; FAUS, M.J. Método Dáder. Guía de Seguimiento Farmacoterapêutico. Granada: UGR, 2003. 43 p.

MINISTÉRIO DA EDUCAÇÃO. Ementa: Institui as Diretrizes Curriculares Nacionais do Curso de Graduação em Farmácia e dá outras providências. Resolução nº 6, de 19 de outubro de 2017. Lex: Diário Oficial da União, Seção 1, p. 30. Brasília, 2017.

MIRANDA, T.M.M.; PETRICCIONE, S.; FERRACINI, F.T.; BORGES FILHO, W.M. Interventions performed by the clinical pharmacist in the emergency department. Einstein. Vol. 10, n. 1, p. 74-85, 2012.

MIYOSHI, E.; BAGLIE, S. Projeto integrado de uso racional de medicamentos e de assistência farmacêutica e médica no CRUTAC. 12 CONEX - Encontro Conversando sobre Extensão. Ponta Grossa, 2014.

OLIVEIRA, A.B.; OYAKAWA, C.N.; MIGUEK, M.D.; ZANIN, S.M.W.; MONTRUCCHIO, D.P. Obstáculos da atenção farmacêutica no Brasil. Revista Brasileira de Ciências Farmacêuticas. Vol. 41, n. 4, out./dez. Universidade Federal do Paraná, 2005.

PIMENTA, P.S. A farmácia escola e suas relações com a sociedade: Uma representação do caso da FAU/UFF. Programa de Pós-graduação em Tecnologia, Centro Federal de Educação Tecnológica Celso Suckow da Fonseca - CEFET/RJ. Rio de Janeiro, 2010.

POSSAGNO, G.C.H.; TOLEDO, A.C.O.; GARABELI, A.A.; MYIOSHI, E.; VEBER, A.P. Atividades desenvolvidas na farmácia escola Prof. Horacio Droppa - UEPG de 2013 a 2015. 14 CONEX - Encontro Conversando sobre Extensão na UEPG. Ponta Grossa, 2016. 
PRUDENTE, L.R.; FERREIRA, T.X.A.M.; BARBOSA, N.L.; DEWULF, N.L.S. A importância da comunicação entre o farmacêutico e o paciente na farmácia comunitária: Relato de caso. $1^{\circ}$ Congresso de Farmácia Comunitária. Universidade Federal de Goiás, Goiânia, 2012.

ROSSIGNOLI, P.; CORRER, C.J., FERNANDEZ-LLIMOS, F. Interés de los alumnos en las actividades de prácticas tuteladas en farmacia escuela en Curitiba-Brasil. Seguimiento Farmacoterapeutico. Vol. 1, n. 2, p. 62 - 68, 2003.

RUSTON, M.D.; SILVA, N.S. A importância da atenção farmacêutica. XII Encontro Latino Americano de Iniciação Científica e VIII Encontro Latino Americano de Pós-Graduação. Universidade do Vale do Paraíba. São Paulo, 2011.

SATURNINO, L.T.M., FERNÁNDEZ-LLIMÓS, F. A Farmácia Escola no Brasil: estado de arte e perspectivas. Revista Brasileira de Farmácia, 2009; Vol. 90, n. 3, p.204-10.

SILVA, E., NAVES, J.O.S., VIDAL, J. O papel do farmacêutico comunitário no aconselhamento ao paciente. Boletim Farmacoterapêutica. Julho/outubro, 2008.

\section{(c)) EY}

Este trabalho está licenciado com uma Licença Creative Commons - Atribuição 4.0 Internacional. 